\title{
Transition Radiation Electron Beam Diagnostic Study At ATF
}

\author{
X.Z. Qiu, X.J. Wang, K. Batchelor and I. Ben-Zvi \\ National Synchrotron Light Source,Brookhaven National Laboratory \\ Upton, N.Y., 11973
}

\section{Abstract}

Recently we have started a program to develop transition rediation based electron beam diagnostics at the Accelerator Test Facility at Brookhaven National Laboratory. In this paper, we will discuss a technique to estimate the the lower limit in electron beam divergence measurement with single foil transition radiation and two-foil transition radiation interferometer. Preliminary experimental data from 4.5 MeV electron beam will be presented.

Introduction

Transition radiation occurs when a charged particle crosses the boundary between two media with different dielectric constants. It was first predicted by Ginzburg and Frank in 1946. [1] The first experimental observation of transition radiation was reported in 1959 by Goldsmith and Jelley. [2] Since then, a significant amount of research have been done on transition radiation both experimentally and theoretically. [3]

Because of the strong dependence of transition radiation on the properties of the radiating particle, it is possible to use transition radiation for charge particle beam diagnostics.

In this paper, we will first review the basic physics of transition radiation from a single foil and a a two-foil interferometer.

We will then estimate the resolution limit when using transition radiation to measure the divergence of a

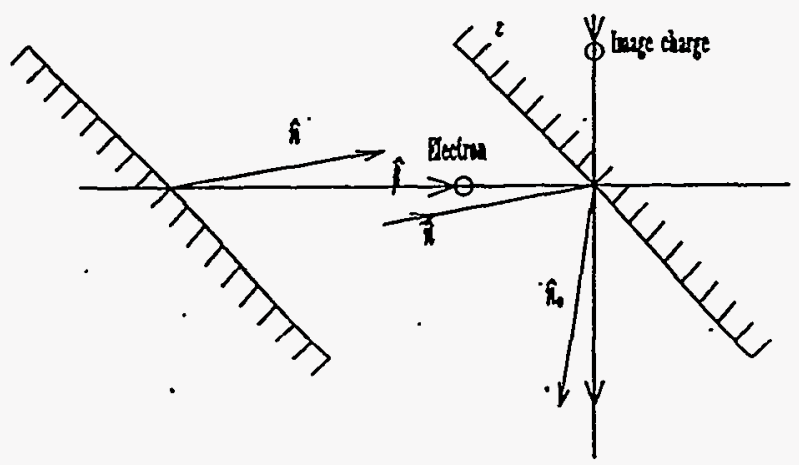

Figure 1: An OTR two-foil interferometer charged particle beam.

Finally, some preliminary result of transition radiation for $4.5 \mathrm{MeV}$ electrons from the BNL/ATF photocathode injector is presented.

\section{Basic theory of optical transition radiation}

Transition radiation occurs when a charged particle crosses the boundary of two medium with different dielectric constant.

When a particle with charged $e$ enters a medium from vacuum at $45^{\circ}$ incident angle, there will be transition radiation around the direction of specular reflection (backward radiation). Assume $|\epsilon-1| \gg \gamma^{-2}$, where $\epsilon$ is the index of refraction of the medium, the angular intensity distribution in the plane of incidence is given by, [4]

$$
\frac{d^{2} I}{d \omega d \Omega}=\frac{e^{2}}{\pi^{2} c} \frac{\theta^{2}}{\left(\gamma^{-2}+\theta^{2}\right)^{2}}
$$

where $\gamma$ is the Lorentz factor and $\theta$ is the angle between the the observation direction and the direction of specular reflection.

In the two-foil transition radiation interferometer configuration as shown in Fig.1.

The forward radiation created when the particle crosses the first foil will interfere with the backward radiation from the second foil, the angular intensity distribution is given by, [4]

$$
\frac{d^{2} I}{d \omega d \Omega}=\frac{e^{2}}{\pi^{2} c} \frac{\theta^{2}}{\left(\gamma^{-2}+\theta^{2}\right)^{2}} \sin ^{2}\left[\frac{\pi L}{2 \lambda}\left(\gamma^{-2}+\theta^{2}\right)\right]
$$

where $L$ is the spacing between the foils.

This manuscript has been authored under contract number DE-AC02-76CH00016 with the U.S. Department of Energy. Accordingly, the U.S. Government retains a non-exclusive, royalty-free license to publish or reproduce the published form of this contribution, or allow others to do so, for U.S. Government purposes. 


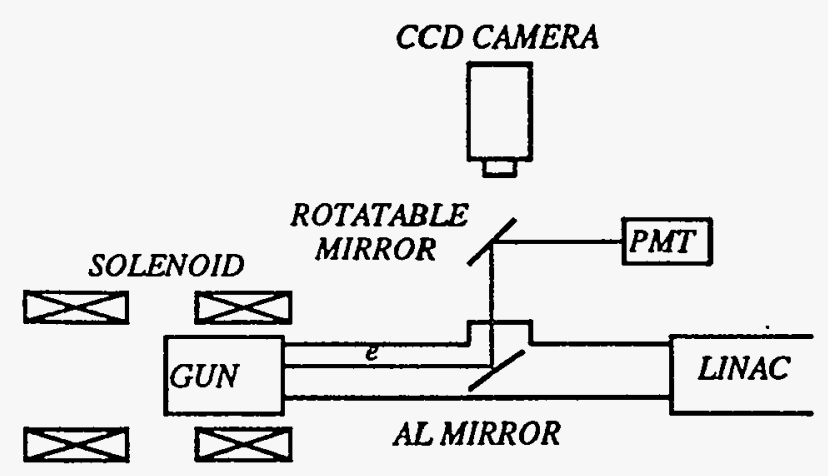

Figure 2: Experimental setup to measure OTR from Low energy electron

If the transition radiation is created by an ensemble of particles, the angular distribution will be smeared. The divergence of the charged particle beam can be measured by looking at the smearing of the distribution. The divergence can also be measured by looking at the smearing of the spectral distribution at a fixed observation angle. This is called transition radiation spectrometer. [3]

Resolution of divergence measurement with transition radiation

The resolution for beam divergence measurement is determined by many factors, for example, the resolution of the detection system and the energy spread of the beam.

For angle $\theta \ll \theta_{m}$, where $\theta_{m}=\gamma^{-1}$, the relative intensity varies as,

$$
\frac{I(\theta)}{I\left(\theta_{m}\right)} \approx \frac{(\theta \gamma)^{2}}{4}
$$

Assume that our detection system has an intensity resolution of $\eta$, then the resolution in divergence due to detection system is,

$$
\theta_{\min } \approx \sqrt{4 \eta} \frac{1}{\gamma}
$$

For a 8-bit detection system,

$$
\theta_{\min } \approx 12.5 \% \frac{1}{\gamma}
$$

The analysis appears to be consistent with the experiment result for single foil transition by Fiorito and Rule. [3] They conclude that beam divergence of the order of $15 \%$ of $\theta_{m} \sim 1 / \gamma$ could be measured.

Similar analysis can be performed for two-foil interferometer. We assume the divergence of the beam is a lot smaller than $1 / \gamma$ and the observation angle is about $1 / \gamma$. We have

$$
\theta_{\min } \approx \sqrt{\eta} \frac{\gamma \lambda}{\pi L}
$$

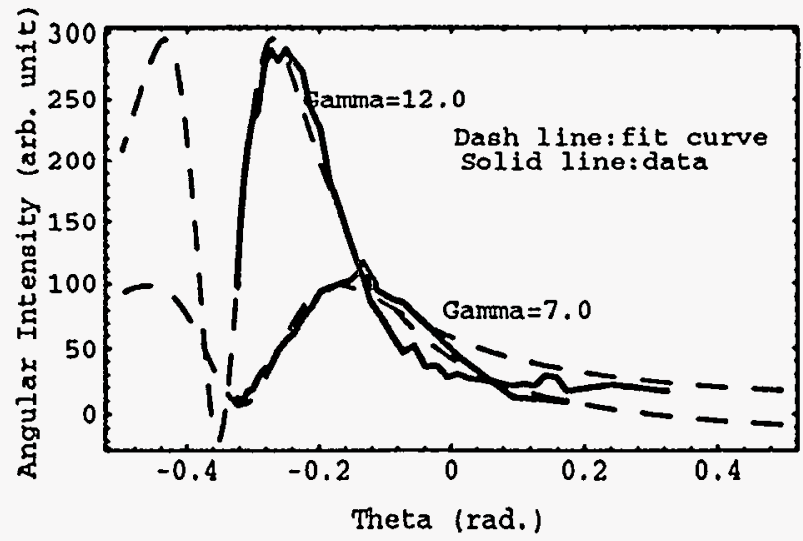

Figure 3: OTR angular distributions with two different RF level of the gun

If we express the inter-foil distance in unit of the formation zone length along the path of the centroid particle,

$$
L=k \frac{\lambda \gamma^{2}}{\pi}
$$

where $k$ is a real number. Then,

$$
\theta_{\min } \approx \frac{\sqrt{\eta}}{k} \frac{1}{\gamma}
$$

In principle, a arbitrarily low divergence can be measured by increasing the inter-foil distance. However, as $\mathrm{L}$ increases, the spacing between interference maxima and minima will decrease and the resolution of the measurement will be limited by angular resolution.

The resolution in divergence for transition radiation spectrometer should be the same as transition radiation interferometer for the same inter-foil spacing.

Caution has to be taken when choosing the first foil. The thickness has to be chosen in such a way that the change in divergence due to scattering of the particles while the beam passing through the foil is a lot smaller than the intrinsic divergence of the beam.

When very thin foils are used, one still tends to estimate the scattering with formulas for multiple scattering. However, when the average number of scatterings for each passing particle $\Omega$ [5] is less than 20, these formulas can no longer be applied. For example, for $1 \mu \mathrm{m}$ thick carbon foil, $\Omega$ is only about 2 . The region in which $20 \leq \Omega \leq 1$ is called plural scattering. Very little literature is available about this region. Further investigation is needed. 


\section{DISCLAIMER}

Portions of this document may be illegible in electronic image products. Images are produced from the best available original document. 


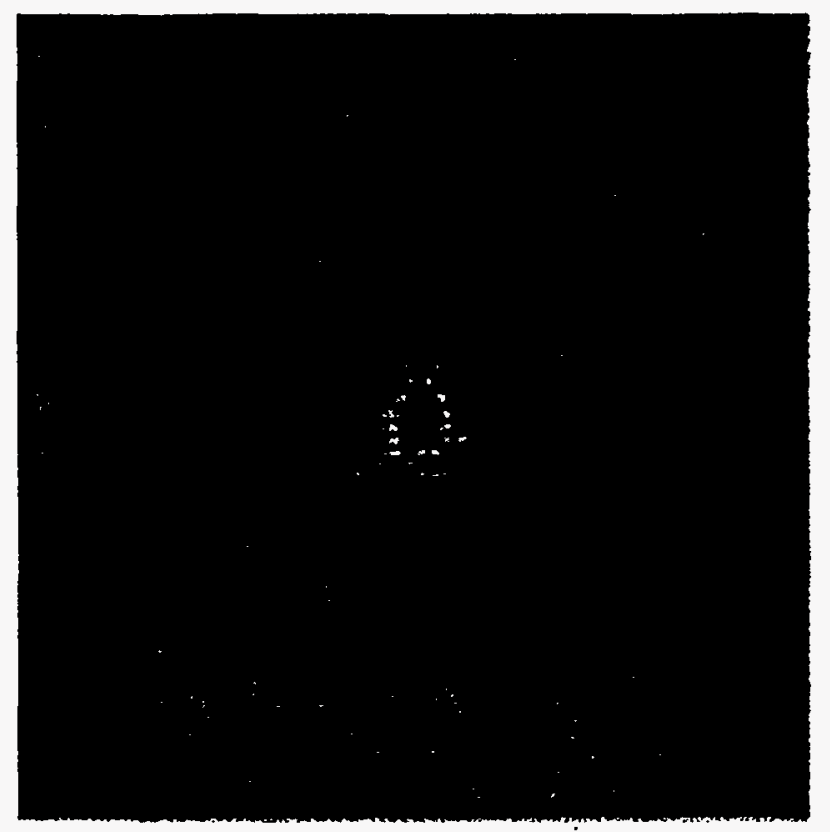

Figure 5: An image of the electron beam obtained from OTR

\section{Experimental result for low energy electron}

The Accelerator Test Facility (ATF) at BNL is a users' facility for experiments in accelerator and beam physics. It is equipped with a high brightness photocathode RFgun, two SLAC linac sections, high-power picosecond pulse lasers. Recently, a new injector was installed to minimize the emittance growth due to spacing charge.

As shown in Fig.2, an aluminum mirror was placed after the electron gun in the path of the electron. A rotatable mirror is placed above the glass window to reflect the transition radiation light into a photo-multiplier tube for detection. The angular distribution of the transition radiation is measured by rotating the mirror.

The angular distributions of transition radiation from electrons produced at two different RF level of the gun is shown in Fig.3. Due to the aperture of the glass window, only one of the two lobes is observed. The data is fitted with (1) to obtain the energy of the electron.

A CCD camera is placed above the window to observe the image of the electron beam. The deflection mirror is rotated to such a position that it would not block the view of the camera. One of the electron beam images is shown in Fig.4. In Fig.5, the density distribution along the vertical and horizontal center lines are plotted.
Horizontal Cursor Psofile
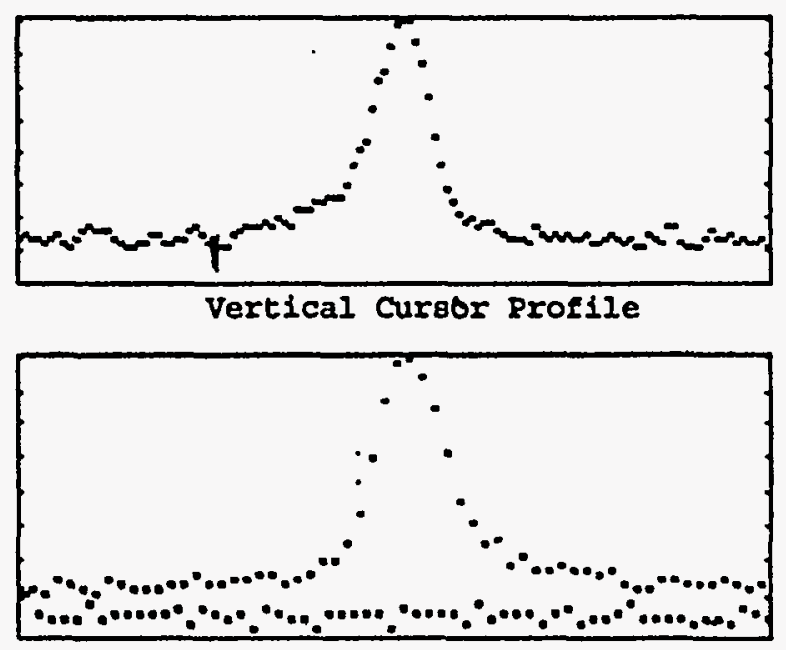

Figure 4: Distribution of the electron beam density along the center lines

\section{Conclusion}

We have estimated the resolution of OTR used to measure the divergence of charged particle beam in terms of the resolution of the detection system. Results of transition radiation from electrons of a few $\mathrm{MeV}$ are presented.

\section{Acknowledgements}

The authors wish to thank Dr. R. Fernow for providing helpful references on the physics of transition radiation and the entire ATF staff for their continuous support.

\section{References}

[1] V.L. Ginzburg and I.M. Frank, Zh. Exp. Teor. Fiz 16, 15 (1946).

[2] P. Goldsmith and J.V. Jelley, Philosophical Magazine 4, 836 (1959).

[3] R. Fiorito and D. Rule, AIP Conference Proceeding, No.319 (1993).

[4] L. Wartski, S. Roland, J. Lasalle, M. Bolore, J. Appl. Phys. 46, 3644 (1975).

[5] G. Lynch and O. Danl, Nucl. Instr. And Meth. B58, 6 (1991).

\section{DISCLAIMER}

This report was prepared as an account of work sponsored by an agency of the United States Government. Neither the United States Government nor any agency thereof, nor any of their employees, makes any warranty, express or implied, or assumes any legal liability or responsibility for the accuracy, completeness, or usefulness of any information, apparatus, product, or process disclosed, or represents that its use would not infringe privately owned rights. Reference herein to any specific commercial product, process, or service by trade name, trademark, manufacturer, or otherwise does not necessarily constitute or imply its endorsement, recommendation, or favoring by the United States Government or any agency thereof. The views and opinions of authors expressed herein do not necessarily state or reflect those of the United States Government or any agency thereof. 